\title{
UMA PESQUISA SOBRE O PERFIL DAS AÇÕES BRASILEIRAS QUE REAGEM À PUBLICAÇÃO DOS RESULTADOS CONTÁBEIS
}

\section{A RESEARCH ABOUT THE PROFILE OF THE BRAZILIAN STOCKS WHICH REACT TO THE PUBLICATION OF ACCOUNTING RESULTS}

\author{
ALFREDO SARLO NETO \\ Doutor em Ciências Contábeis pela \\ Universidade de São Paulo \\ Professor Adjunto do Centro de Ciências Jurídicas e \\ Econômicas da Universidade Federal do Espírito Santo \\ Vitória/ES, Brasil \\ E-mail: asarlo@uol.com.br
}

\author{
FERNANDO CAIO GALDI \\ Doutor em Ciências Contábeis pela \\ Universidade de São Paulo \\ Professor Associado da Fundação Instituto Capixaba de \\ Pesquisa em Contabilidade, Economia e Finanças \\ Vitória/ES, Brasil \\ E-mail: fernando.galdi@fucape.br
}

FLÁVIA ZÓBOLI DALMÁCIO

Doutoranda em Ciências Contábeis pela Universidade de São Paulo

Professora da Fundação Instituto Capixaba de

Pesquisa em Contabilidade, Economia e Finanças

Vitória/ES, Brasil

E-mail: flavia@fucape.br

\section{Resumo}

Este trabalho estuda a influência de algumas características sobre a reação dos preços das ações à divulgação das informações contábeis. A pesquisa realizada procura verificar se a média dos retornos anormais das ações são sensibilizadas pelas seguintes características: tipo de ação, regulamentação, controle acionário e liquidez. No seu desenvolvimento, foi utilizada como metodologia a técnica estatística MANOVA. A amostra pesquisada foi composta por ações negociadas na Bolsa de Valores de São Paulo (BOVESPA), durante o período de 1995 a 2002. Os resultados encontrados indicam que as médias dos retornos anormais são sensíveis às características controle acionário e liquidez. Porém, as evidências encontradas devem ser analisadas com restrições, pois a amostra estudada não atingiu alguns pressupostos exigidos pela técnica estatística utilizada (MANOVA). A partir dos resultados encontrados pode-se sugerir algumas propostas para novas pesquisas, na tentativa de buscar novas evidências sobre a influência das características dos mercados sobre a reação dos preços à divulgação da informação contábil. Entre as sugestões, encontram-se: a ampliação da janela do evento para analisar a influência de características sobre períodos superiores a três meses; a verificação de outras características, além das estudadas nesta pesquisa e a aplicação de outras técnicas estatísticas, tais como análise de cluster ou regressão logística.

Palavras-chave: relevância da contabilidade; retorno anormal; MANOVA.

\begin{abstract}
This work studies the influence of some characteristics on the reaction of stocks prices to the disclosure of accounting information. The research seeks to verify whether the average of the abnormal returns of the stocks is sensitive to the following characteristics: kind of stock, rules, stock control and liquidity. It was used as methodology MANOVA statistics technique. The analyzed sample was composed by stocks negotiated in BOVESPA (São Paulo Stock Market), in the period from 1995 to 2002. The results indicate that the average of abnormal returns is sensitive to stock control and liquidity. However, the found evidence must be analyzed under restrictions as the studied sample did not reach some assumptions demanded by the used statistical technique (MANOVA). Based on the found results, some proposals for new research can appear, in order to find fresh evidence about the influence of market characteristics over the reaction of prices to the accounting information disclosure. Among the suggestions, there are: the expansion of the event window, in order to analyze the influence of the characteristics on periods larger than three months; the study of other characteristics, beyond the ones studied by this research and the application of other statistic techniques, such as the cluster and the logistic regression.
\end{abstract}

Key words: relevance of Accounting, abnormal returns, MANOVA. 


\section{INTRODUÇÃO}

Recentemente, a pesquisa em contabilidade no Brasil vem realizando diversos trabalhos dedicados a estudar a relação entre o lucro contábil e os preços das ações. Devido a sua relevância para a pesquisa positiva em contabilidade, a relação entre o lucro contábil e os preços das ações foi extensamente estudada nos mercados desenvolvidos.

Nos trabalhos realizados pela literatura brasileira foram encontradas evidências indicando que os preços reagem à divulgação das informações contábeis.Entretanto, observou-se que grupos de ações com determinadas características reagem de forma diferente, tanto em magnitude, como na direção.

Entre os diversos trabalhos realizados, deve-se destacar ${ }^{i}$ Terra (1993), Sarlo Neto (2005), Lima (2004), Lopes (2002), e Sarlo Neto (2004). Nestes trabalhos foi observada a influência das mais diversas características sobre a reação dos preços a divulgação das informações contábeis, tais como: tipos de ações, regulamentação, controle acionário e liquidez.

A partir das evidências encontradas, pode-se levantar a hipótese de que a reação às informações contábeis possa estar vinculada à ocorrência de uma determinada característica ou de um conjunto delas. Nesse sentido, algumas questões interessantes para a contabilidade podem ser estruturadas, tais como: a reação à informação contábil está relacionada com alguma característica específica da ação? A reação depende de característica da ação? A reação vinculada a alguma característica da ação representa uma anomalia do mercado brasileiro?

Este trabalho não tem a pretensão de esclarecer todas as questões relacionadas ao tema, mas tem, de forma específica, a finalidade de verificar se a reação à divulgação das informações contábeis está relacionada a uma determinada característica ou a um conjunto de características comuns pertencentes às ações brasileiras.

Assim, sobre o conjunto de evidências encontradas pela literatura realizada, lançase a seguinte questão de pesquisa: os preços de grupos de ações com características diferentes reagem diferentemente à divulgação da informação contábil?

Espera-se que esta pesquisa venha a contribuir para a ampliação dos conhecimentos sobre a relevância da informação contábil para o mercado de capitais, adicionando conhecimentos sobre a influência de elementos externos ao ambiente contábil sobre a reação dos preços à divulgação da informação contábil.

\section{A REAÇÃO DOS PREÇOS DAS AÇÕES À DIVULGAÇÃO DOS LUCROS CONTÁBEIS}

De acordo com a Teoria da Hipótese do Mercado Eficiente, na forma semi-forte, as informações contábeis deveriam estar refletidas nos preços das ações. Desta maneira, qualquer variação nos lucros contábeis provocaria uma variação nos preços das ações.

Um mercado é dito eficiente no sentido semi-forte quando os preços refletem (incorporam) toda informação publicamente disponível, incluindo informações tais como demonstrações financeiras publicadas pela empresa, bem como dados históricos de preço (ROSS, 1995, p.267). 
Num cenário, em que o mercado é eficiente na forma semi-forte, as informações geradas pela Contabilidade deveriam estar refletidas nos preços das ações. Então, nessa situação, haveria uma forte relação entre a Contabilidade e o mercado, de forma que a alteração em uma variável contábil provocaria a variação dos preços das ações.

Muitas pesquisas realizadas constataram que a divulgação dos lucros contábeis impacta os preços das ações, porém nos resultados encontrados não foi observada uma correlação prefeita. As evidências encontradas indicam que os preços seguem a mesma direção dos lucros, mas não variam com a mesma escala.

Entretanto, os resultados apresentados nessa pesquisa e em muitas outras que a sucederam não apresentaram correlação perfeita (igual a 1) entre o lucro e o preço das ações. As evidências encontradas assinalam que, apesar de seguirem a mesma direção, lucros e preços apresentam variações com intensidades diferentes, ou seja, as variações positivas nos lucros estão relacionadas com variações positivas nos preços, mas não com a mesma escala de variação.

Parte do motivo para a falta de correlação perfeita está no fato de que os preços captam o impacto de um conjunto de informações muito mais amplo do que o representado apenas por lucros contábeis. Por exemplo, os preços reagem a coisas tão exóticas como boatos de guerra e tão corriqueiras quanto variações da taxa de juros de Letras do Tesouro. (HENDRIKSEN e VAN BREDA, 1999, p. 206)

Em estudos complementares, foram encontradas evidências de influência dos mais diversos elementos, presentes no ambiente econômico ou no ambiente de governança corporativa, sobre a relação entre os lucros e preços das ações.

As evidências encontradas servem como suporte para construções de hipóteses de pesquisa sobre as influências dos mais diversos tipos de elementos quanto à relação entre lucros e preços.

\section{AS CARACTERÍSTICAS DAS AÇÕES E A REAÇÃO DOS PREÇOS À DIVULGAÇÃO DOS LUCROS}

As evidências encontradas pela literatura indicam que diversos elementos influenciam na intensidade da reação dos preços à divulgação das informações contábeis.

Entre esses diversos elementos podem ser considerados aqueles presentes no ambiente econômico, como o nível das taxas de juros e o nível de liquidez do mercado, ou aqueles presentes no ambiente governamental ou societário da Contabilidade, como a regulamentação ou a classificação das ações negociadas.

As pesquisas realizadas nos mercados desenvolvidos foram encontradas evidências sobre a influência de diversos elementos sobre a relação entre lucro e preços. Nos mercados desenvolvidos, a hipótese sobre a influência desses elementos sobre a relação entre lucro e os preços foi amplamente estudada. Entre eles, podem-se destacar as seguintes evidências: Grant (1980) encontrou evidências de que o efeito tamanho da empresa não impacta nessa relação; Ball, Kothari e Watts (1993) encontraram evidências sobre a influência da mudança do risco da empresa; Park e Pincus (1997) confirmaram a hipótese sobre a influência do endividamento. 
Em mercados menos desenvolvidos, como a Espanha, Pellicer e Rees (1999) encontraram evidências sobre a influência da regulamentação. No México, Davis-Friday e Rivera (2000) verificaram a influência da inflação. Na Itália, Carnestrelli e Ziemba (2000) encontraram evidências do "efeito final do período" na bolsa de valores de Milão. Na bolsa de valores de Tóquio, no Japão, Comolli e Ziemba (2000) encontraram evidências do efeito final de período da mesma forma que nos Estados Unidos. Muradoglu (2000) fornece uma discussão extensiva da bolsa de valores de Istambul e demonstra que muitas das anomalias encontradas nos mercados mais desenvolvidos são comuns ao mercado turco. As mesmas anomalias são encontradas em Helsinque de acordo com Martikainen (2000). Patel (2000) fornece fortes evidências do "efeito tamanho" em 22 mercados emergentes (incluindo o Brasil) classificados de acordo com o International Finance Corporation Global Index. No entanto, esses resultados não são correlacionados entre os 22 mercados analisados da forma que Hawawini e Keim (2000) apresentam para os mercados desenvolvidos. As mesmas anomalias são encontradas na Asia de acordo com Koh e Wong (2000).

No mercado brasileiro, Terra (1993) pesquisou o valor de mercado do controle a partir de evidências sobre os custos e benefícios dos direitos de voto das ações, Lopes (2001) analisou a influência dos intangíveis, Guimarães e Ness (2002) pesquisaram diversos fatores que pudessem influenciar a diferença de preços entre ações ordinárias e preferenciais, Sarlo Neto (2002) investigou a influência do excesso da regulamentação da Contabilidade sobre os setores financeiro e elétrico, Lima (2004) verificou o lançamento de ADR's, Sarlo Neto (2004) pesquisou diversos fatores, tipos de ações, tipo de controle, regulamentação e liquidez. Recentemente, Sarlo Neto (2005) apresentou diferenças de reação entre os tipos diferentes de ações (ordinária versus preferenciais), Mirian (2005) estudou o impacto dos anúncios e lançamentos de ADR's e Dilza (2005) encontrou evidências sobre o efeito contágio.

Na maioria dos trabalhos realizados no Brasil, foram encontradas evidências de que as características estão relacionadas com a magnitude da reação dos preços à divulgação das informações contábeis. Como exemplo, segue a pesquisa elaborada por Sarlo Neto (2005). Nesse trabalho, o autor encontrou diferenças nas reações apresentadas pelas ações de tipos diferentes (preferenciais e ordinárias).
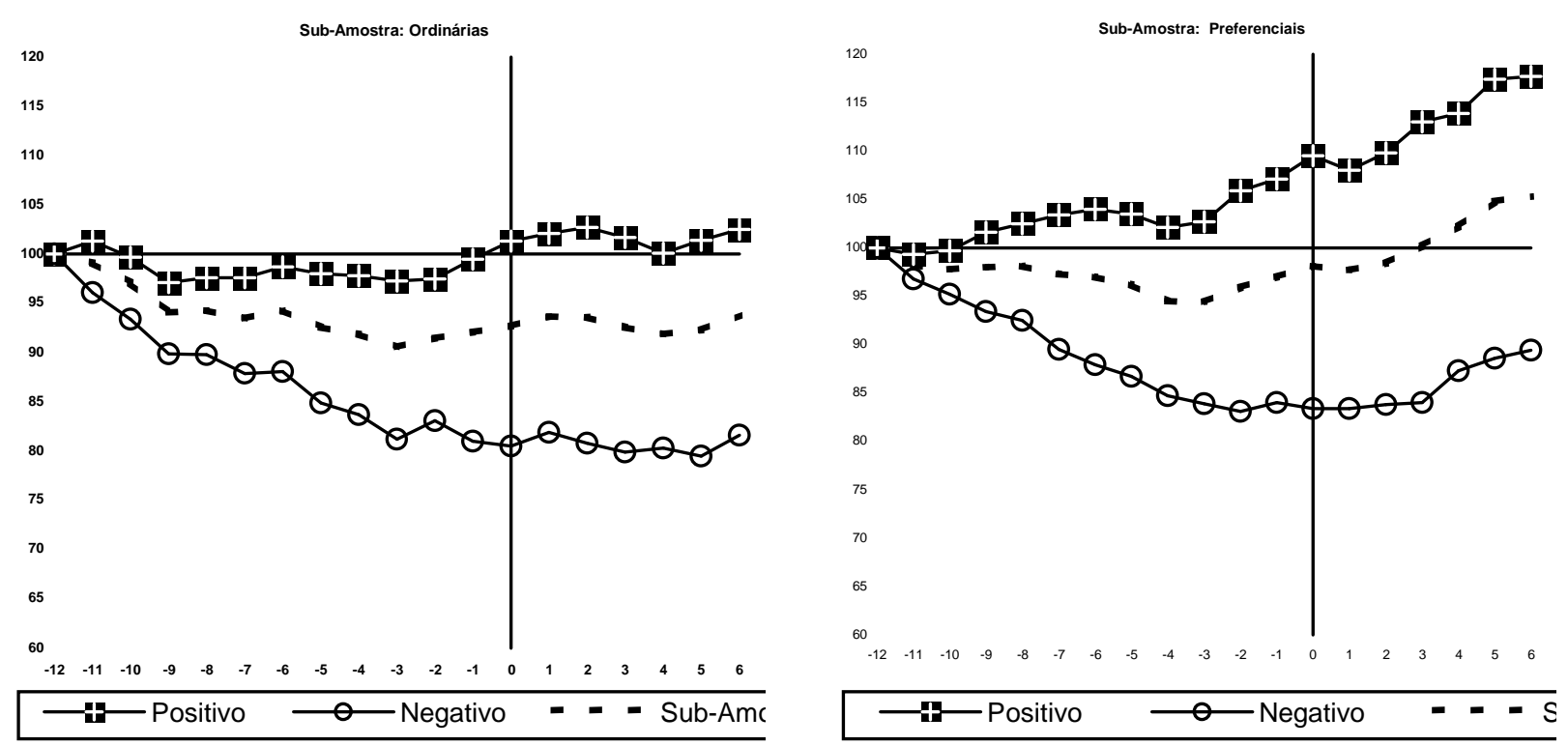

Gráfico 1: Relação entre características e reação à informação contábil 
Conforme os gráficos acima, observa-se que os retornos (positivos e negativos) das ações preferenciais variam de acordo com os lucros anormais. No caso das ações ordinárias, somente os retornos negativos reagem aos lucros anormais.

As evidências encontradas, não somente neste trabalho, mas como em todo o conjunto de trabalhos dedicados a estudar a relevância das informações contábeis indicam que as reações dos preços estão relacionadas a algum tipo de característica das ações.

\section{HIPÓTESES}

Considerando as evidências encontradas no mercado brasileiro, que indicam a influência de vários elementos sobre a reação dos preços à divulgação da informação contábil, pode-se estruturar uma associação entre as características das ações e as suas respectivas reações à divulgação das informações contábeis.

De forma específica, este estudo adotará como referência (pressuposto) as evidências encontradas por Sarlo Neto (2004). Neste estudo (dissertação), o autor encontrou diferenças estatísticas entre os retornos anormais de empresa com atributos opostos em relação às seguintes características: Tipo de Ações, Nível de Regulamentação, Tipo de Controle e Liquidez. A partir destes resultados, pode-se estruturar, conforme a Figura 1, a relação entre as características citadas e as reações das ações à divulgação dos resultados contábeis.
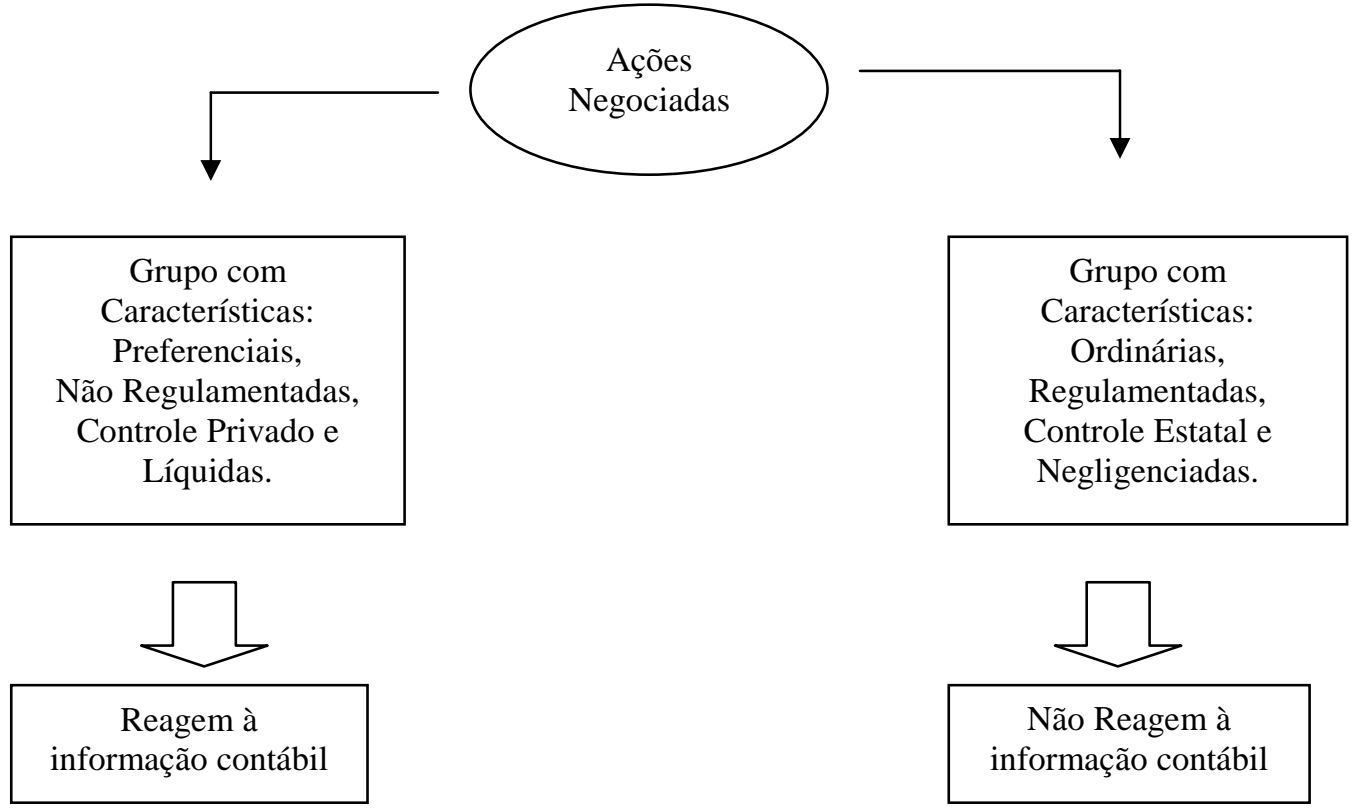

Figura 1: Relação entre características e reação à informação contábil

De acordo com relação estruturada, em que grupos de ações com determinadas características reagem diferentemente, na forma e em magnitude, à divulgação da informação contábil, de grupos com outras características, lança-se a seguinte hipótese de pesquisa:

$\mathrm{H}_{0}$ : As médias dos retornos anormais em torno da divulgaçãa das informações contábeis são diferentes para grupos de ações que não possuem as mesmas características. 
A hipótese elaborada procura verificar se grupos de ações com características diferentes não reagem de forma similar em um intervalo de tempo em torno da data de divulgação das informações contábeis.

Pela forma como foi elaborada a hipótese, se for observado que ações que possuem características diferentes não reagem da mesma forma à divulgação das informações, haverá a verificação de que as reações estão relacionadas às características das ações.

\section{METODOLOGIA}

Para testar a hipótese formulada, foi necessária a utilização de diversas técnicas para analisar a relação das variáveis envolvidas na pesquisa. O desenvolvimento empírico da pesquisa ocorrerá em duas etapas, por meio da aplicação de técnicas estatísticas distintas.

Num primeiro momento, haverá o cálculo da reação das ações em relação à divulgação dos lucros contábeis. A metodologia adotada é similar aos outros trabalhos realizados sobre o tema, tais como o seminal trabalho realizado por Ball e Brown (1968). Essa metodologia consiste na apuração do retorno anormal acumulado durante o período de observação (janela) em torno da data de divulgação das informações contábeis.

No momento seguinte, será aplicada a técnica multivariada MANOVA, com a intenção de analisar a influência das características das ações sobre a média dos retornos acumulados. De forma a observar a influência das características em torno do momento da divulgação, serão analisadas as variáveis envolvidas na seguinte janela.

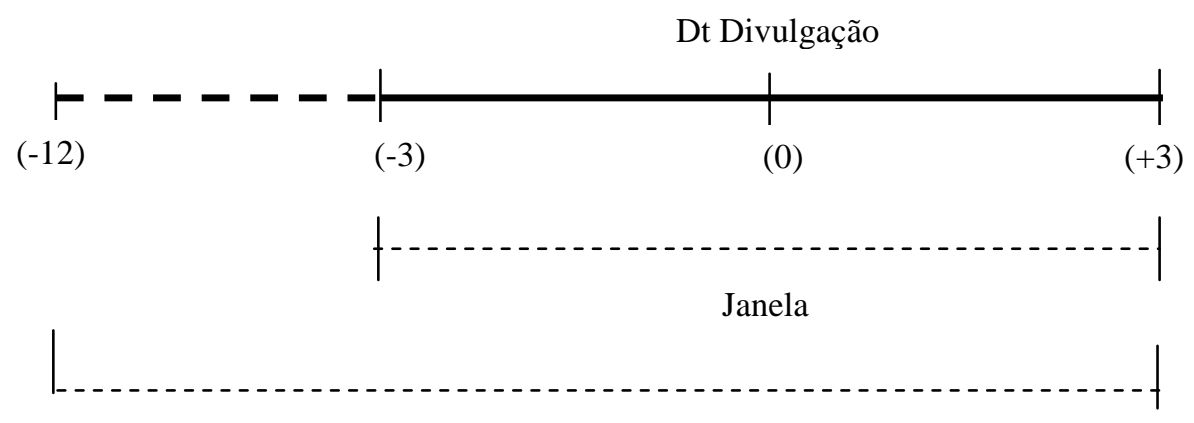

Retorno Anormal Acumulado

Figura 2: Intervalos da janela do evento

Como data do evento, também chamada de data (0), foi considerada a data de divulgação $0^{\text {ii }}$ dos resultados contábeis anuais. 0 período pré-evento engloba os meses de (3 ) a (0), sendo o período pós-evento formado pelos meses $(0)$ a $(+3)$. De forma integral, a amplitude do evento é de 6 meses, com início três meses $(-3)$ antes do evento $(0)$ e com término três meses após $(+3)$.

Pela amplitude definida para o evento, nota-se que a pesquisa parte da premissa de que a divulgação dos lucros anuais é considerada como uma forte sinalização sobre as expectativas futuras de uma empresa. A pesquisa utiliza a divulgação das informações contábeis para o mercado como evento potencial para inferir uma variação nos preços das ações. Ao examinar a variação dos preços em torno (antes, durante e depois) do evento, 
pode-se conhecer a atitude dos investidores em virtude da divulgação das informações contábeis.

\subsection{CÁLCULO DOS RETORNOS ANORMAIS}

\subsubsection{Retorno Anormal Acumulado (CAR)}

o retorno anormal acumulado (CAR) de cada ação é calculado a partir da capitalização dos retornos anormais ${ }^{\mathrm{iii}}$ mensais apurados até uma determinada data t. Sendo a amplitude do evento igual a 16 meses, com início no mês -12 e final no mês +6 , tem-se para o cálculo do retorno anormal acumulado (CAR) a seguinte equação:

$$
C A R_{i t}=\prod_{t=-11}^{T}\left(1+R A_{i t}\right)-1 \quad T=-11,-10, \ldots,+,+5,+6
$$

Em que: $C A R=$ Retorno Anormal Acumulado para empresa $i$, e $R A=$ Retorno Anormal para a empresa $i$ no período $t$.

0 índice de retorno acumulado demonstra o comportamento da ação durante a janela do evento. Por meio desse índice, torna-se possível acompanhar a reação da ação antes, durante e depois da divulgação das informações contábeis.

\subsubsection{Retorno Anormal Acumulado ao Quadrado (CARq)}

De maneira a facilitar a funcionalidade da pesquisa e a ajustar os dados para a utilização correta da técnica estatística MANOVA, foi implementado um ajuste no cálculo do retorno anormal acumulado.

Levando em consideração que o estudo está direcionado na magnitude e não na direção da reação, para facilitar a aplicação e o desenvolvimento da metodologia escolhida (MANOVA), os retornos anormais foram elevados ao quadrado tornando todas as variações, positivas ou negativas, em variações de sinal positivo. Assim, o cálculo do retorno anormal acumulado foi ampliado para a seguinte formulação:

$$
C A R q_{i t}=\left(\prod_{t=-11}^{T}\left(1+R A_{i t}\right)-1\right)^{2} \quad T=-3,0,+3
$$

Em que: $C A R=$ Retorno Anormal Acumulado ao Quadrado para empresa $i$, e $R A=$ Retorno Anormal para a empresa $i$ no período $t$.

\subsection{ANÁLISE MULTIVARIADA DE VARIÂNCIA - MANOVA}

Num segundo momento, de posse dos retornos anormais acumulados, seria aplicada a técnica multivariada MANOVA, ${ }^{\text {iv }}$ pela qual seriam relacionados os retornos para cada data escolhida $(-3,0,+3)$ e as variáveis categóricas selecionadas de acordo com a hipótese de trabalho elaborada.

MANOVA é uma técnica de dependência que compara as diferenças de médias para duas ou mais variáveis dependentes métricas, com base em um conjunto de variáveis independentes categóricas (não-métricas). (WASSERMAN, 2005) 
Com base nas variáveis (características escolhidas), o modelo a ser testado pela MANOVA possui a seguinte estrutura:

$$
\left(C A R q^{-3}, C A R q^{0}, C A R q^{+3}\right)=f(T, R, C, L)
$$

Em que: $C A R q^{n}=$ Retorno Anormal Acumulado ao Quadrado no período $\mathrm{n}$, e $T=$ tipo de ação, $R=$ nível de regulamentação, $C=$ Controle Acionário e $L=$ Liquidez.

Conforme o modelo apresentado, as variáveis independentes correspondem às características selecionadas pela hipótese de pesquisa e que podem ser classificadas como categóricas com as seguintes segregações: Tipo de ação (Preferencial versus Ordinária), Nível de Regulamentação (Regulamentadas versus Não Regulamentadas), Tipo de Controle Acionário (Privado versus Estatal) e Liquidez (Mais Liquidas versus Menos Líquidas). Como variáveis dependentes, se encontram os retornos anormais para cada período de observação da janela.

Conforme o modelo estruturado, procura-se por meio do Teste MANOVA verificar se as diversas categorias das variáveis independentes ( $T, R, C$ e $L$ ) influenciam as médias dos retornos anormais em cada uma das datas do evento $(-3,0,+3)$.

\section{SELEÇÃO DE DADOS}

Foram utilizadas neste estudo as ações negociadas na Bolsa de Valores de São Paulo - BOVESPA, sendo que as informações sobre as empresas pesquisadas foram obtidas por intermédio do banco de dados disponibilizado pela Economática.

A pesquisa analisou o período após o Plano Real, contemplando os exercícios contábeis de 1995 a 2001. Considerando-se que os eventos (data de divulgação) ocorrem após o encerramento do exercício, e que os retornos anormais foram calculados com base em parâmetros mensurados com base em 60 meses anteriores, o período integral para coleta e análise de dados foi de $1^{\circ}$ de janeiro de 1990 a 12 de dezembro de 2002 . Para atender às necessidades da pesquisa, foram coletados os seguintes dados:

- Resultado Contábil: foram considerados os resultados contábeis anuais, apurados durante os 12 meses de cada exercício (01 de janeiro a 31 de dezembro);

- Data do Evento: foi considerada a data de divulgação ${ }^{\text {vi }}$ das publicações financeiras, o dia em que as informações contábeis foram disponibilizadas aos acionistas e a outros interessados;

- Lucro Contábil: foi utilizada a forma Lucro por Ação (LPA);

- Preço dos Ativos: consideraram as cotações diárias de fechamento ajustadas por proventos; ${ }^{\text {vi }}$

- Índice de Mercado: foi selecionado o IBOVESPA, índice calculado e divulgado pela BOVESPA (Bolsa de valores de São Paulo);

- Setor Econômico: foi utilizada, para cada empresa, a respectiva classificação setorial previamente existente e disponibilizada no banco de dados utilizado (Economática);

- Principal Acionista: foi levantado o acionista com maior participação no capital votante de cada empresa no dia 31 de março de cada ano; 
Os dados coletados na pesquisa estão em moeda ajustada pela inflação (IGP-DI), e foram selecionadas todas as ações, ordinárias ou preferências, que contemplassem os seguintes critérios de seleção:

- Apresentassem cotação vii em todos os meses compreendidos pelo período analisado (janeiro/1990 a Dezembro de 2002); e

- Apresentassem os resultados contábeis e respectivas datas de divulgação para os exercícios compreendidos pelo período analisado.

Por meio dos critérios de seleção apresentados e a partir da população de todas as ações negociadas na BOVESPA, durante o período de pesquisa, foi obtida uma amostra de 108 ações, sendo 25 ordinárias e 83 preferenciais, de 93 empresas diferentes (Apêndice A).

De posse dos dados selecionados, houve a aplicação da metodologia indicada gerando um conjunto de eventos. Por intermédio das ações e correspondentes lucros por ação selecionados foi gerado um total de 756 eventos para o período analisado.

No interesse de manter o rigor científico deste estudo, foram excluídos da amostra todos os eventos que não atenderam na data 0 (data de divulgação) às seguintes restrições:

- Valores de $B$ significantes para um nível de $10 \%$, e

- Ausência de autocorrelação ${ }^{\text {viii }}$ dos resíduos.

Após a exclusão dos eventos pelas restrições ${ }^{\mathrm{ix}}$ impostas, a amostra final, denominada, a partir desse momento, de amostra do mercado, acabou sendo formada por um total de 559 eventos.

\section{CLASSIFICAÇÃO DAS SUB-AMOSTRAS DE RETORNOS ANORMAIS ACUMULADOS}

De posse dos dados selecionados, houve a aplicação da metodologia indicada promovendo a geração de um conjunto de retornos anormais acumulados. Por intermédio das ações e correspondentes lucros por ação selecionados, foram gerados um total de 756 eventos para cada data de observação $(-3,0,+3)$ presente na janela de observação.

Após a exclusão dos eventos que não atenderam aos atributos de inclusão (descritos no item 6), a amostra final, chamada a partir deste momento de amostra "mercado", acabou sendo constituída por um total 559 eventos de 91 empresas diferentes.

Para atender aos objetivos da pesquisa, o conjunto de eventos que compõem a amostra do mercado foram posteriormente segregados e agrupados em diferentes subamostras. A amostra do mercado foi dividida em função das características do mercado brasileiro discutidas na formulação das hipóteses (item $\mathrm{n}^{\circ} 4$ ). Assim, a partir da amostra de mercado foram criadas um total de 8 (oito) sub-amostras. 


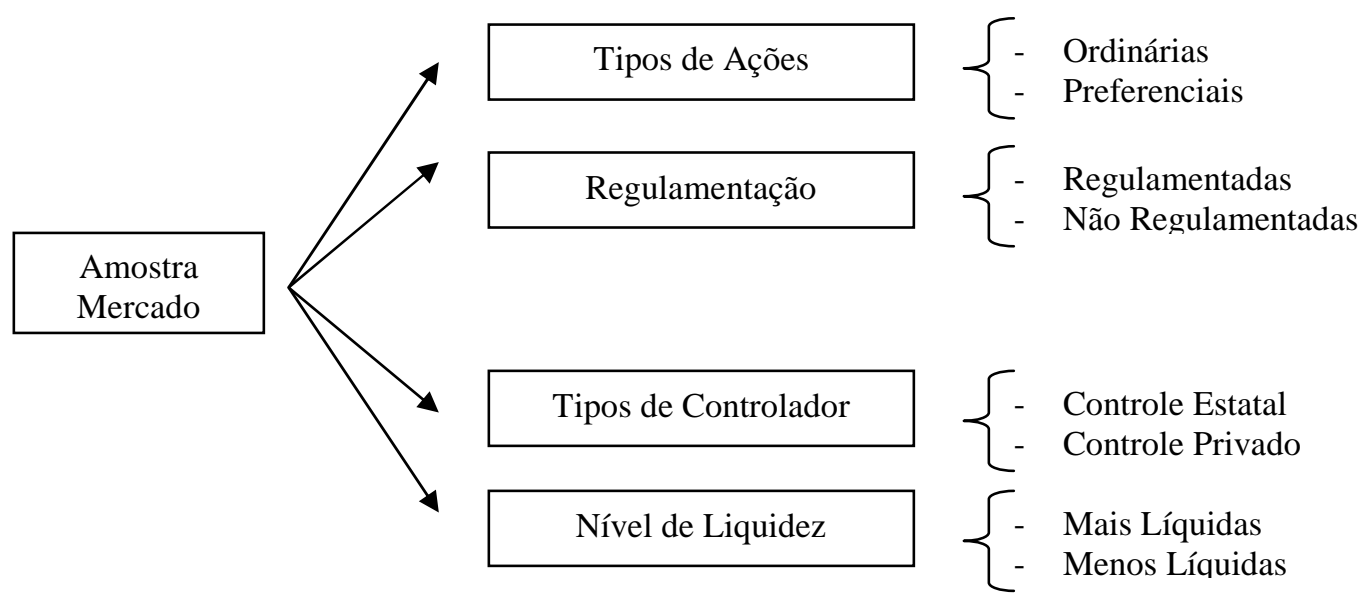

Figura 3: Sub-Amostras segregadas conforme características do mercado brasileiro

Conforme a Figura 3, de posse da amostra de mercado, os eventos foram classificados e separados, em grupos não excludentes, conforme o tipo de ação (ordinária ou preferencial), regulamentação (Regulamentadas e Não Regulamentadas), tipos de controle (Estatal e Privado) e nível de liquidez (mais líquidas e menos líquidas). Com base na classificação proposta, as sub-amostras tiveram a seguinte composição de eventos:

Tabela 1: Número de ações por Sub-amostras

\begin{tabular}{|c|c|c|}
\hline \multirow{2}{*}{ Sub-amostras } & Características & Total \\
\hline \multirow{2}{*}{ Tipo } & Ordinárias & 127 \\
\cline { 2 - 3 } & Preferenciais & 389 \\
\hline \multirow{2}{*}{ Regulamentação } & Não Regulamentada & 424 \\
\cline { 2 - 3 } & Regulamentada & 92 \\
\hline \multirow{2}{*}{ Controle } & Estatal & 72 \\
\cline { 2 - 3 } & Privado & 444 \\
\hline \multirow{2}{*}{ Liquidez } & IBOVESPA & 169 \\
\cline { 2 - 3 } & Não IBOVESPA & 347 \\
\hline
\end{tabular}


Com base na composição de cada sub-amostras, segue no gráfico abaixo a evolução das médias dos retornos anormais acumulados ao quadrado (CARq) para cada uma das datas da janela de observação:

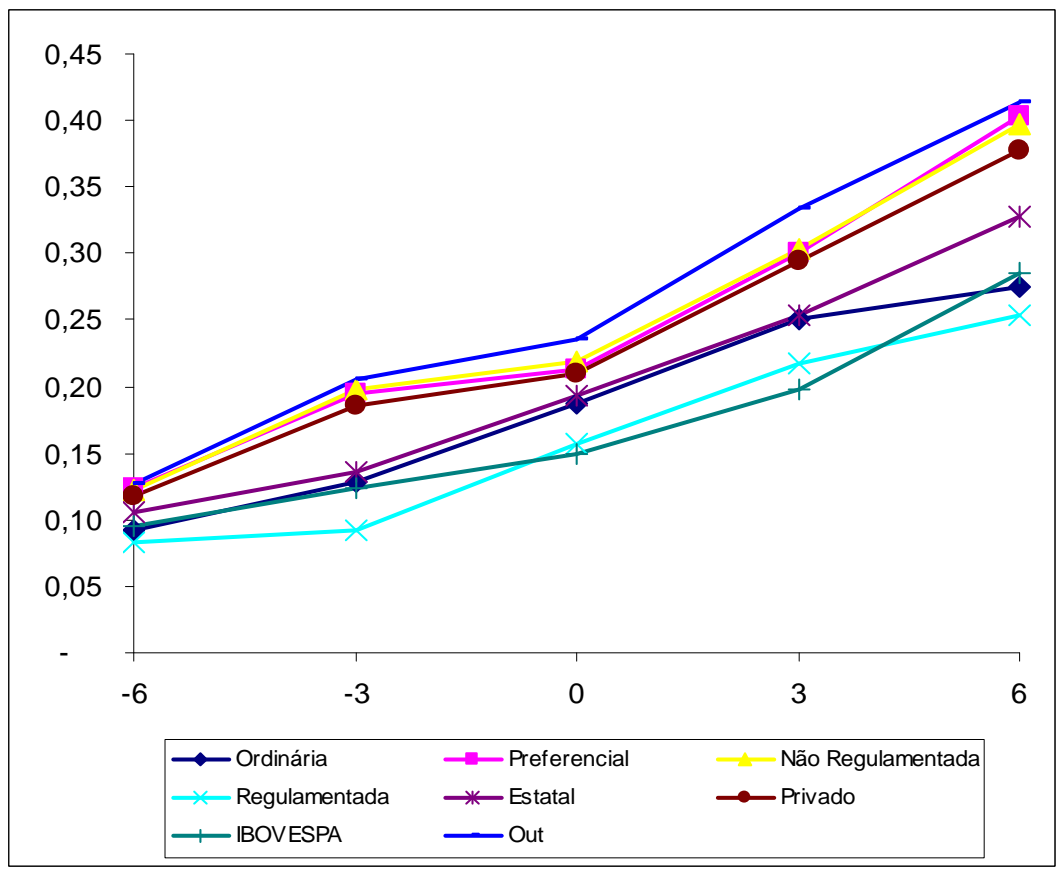

Gráfico 2: Retornos Anormais Acumulados por Data e por Sub-Amostra

Por meio das evoluções representadas no gráfico, observa-se que as sub-amostras apresentaram médias de retornos anormais acumulados diferentes entre si. A representação gráfica é uma indicação de que grupos de ações com características diferentes não reagem com a mesma magnitude à divulgação da informação contábil.

Entretanto, a representação gráfica é apenas visual, não significando que as subamostras registraram reações estatisticamente diferentes. Nesse sentido, torna-se relevante a aplicação da técnica multivariada MANOVA, de forma a verificar se as médias dos retornos anormais acumulados são influenciadas por algumas das características selecionadas pela pesquisa.

\section{RESULTADOS ENCONTRADOS}

Considerando-se a aplicação do teste MANOVA sobre os dados selecionados, procura-se verificar se as categorias das ações possuem a capacidade de sensibilizar as médias dos retornos anormais acumulados.

Os resultados serão apresentados em duas etapas. A primeira etapa compreende o teste de igualdade das médias dos retornos anormais acumulados ao longo dos grupos de ações formados de acordo com cada uma das categorias. Sendo a segunda etapa destinada à análise da influência específica de cada categoria sobre as médias de cada data de observação do evento. 
Antes da aplicação do teste MANOVA, procurou-se verificar se as variáveis atendem ao pressuposto de igualdade da matriz de variância. Para esta verificação foi aplicado o teste Box'M, no qual se deseja rejeitar a hipótese nula.

Pelos resultados do Teste Box'M, não houve a rejeição da Hipótese Nula. Apesar da exigência desta rejeição, procurou-se relaxar este pressuposto devido à sensibilidade do teste a amostras com elevado número de observações, como é o caso deste estudo que trabalha com um total de 6.708 (559 retornos acumulados $\times 4$ características $\times 3$ datas) observações.

\subsection{TESTE DE IGUALDADE DAS MÉDIAS ENTRE OS GRUPOS:}

Nas tabelas 2, 3 e 4, são apresentados os resultados dos testes sobre a igualdade das médias dos retornos anormais ao longo das datas de observação $(-3,0,+3)$ do evento. Nesta verificação a rejeição da hipótese nula, de igualdade das médias, representa que as médias dos retornos anormais são sensíveis a determinado tratamento (categoria).

Tabela 2: Significância das Categorias

\begin{tabular}{|c|c|c|c|c|c|}
\hline Categoria & Teste & Value & $\mathbf{F}$ & Sig. & Obs. Power \\
\hline \multirow[t]{4}{*}{ TIPO } & Pillai's Trace & ,002 &, 270 & ,847 & , 102 \\
\hline & Wilks' Lambda & ,998 & ,270 & ,847 & , 102 \\
\hline & Hotelling's Trace & ,002 &, 270 & ,847 & , 102 \\
\hline & Roy's Largest Root & ,002 & ,270 & ,847 &, 102 \\
\hline \multirow[t]{4}{*}{ REGULA } & Pillai's Trace & ,002 &, 404 & ,750 & , 130 \\
\hline & Wilks' Lambda & ,998 & ,404 & ,750 &, 130 \\
\hline & Hotelling's Trace & ,002 & ,404 & ,750 &, 130 \\
\hline & Roy's Largest Root & ,002 & ,404 & ,750 & ,130 \\
\hline \multirow[t]{4}{*}{ CONTROLE } & Pillai's Trace & ,023 & 3,955 & ,008 & ,832 \\
\hline & Wilks' Lambda & ,977 & 3,955 & ,008 & ,832 \\
\hline & Hotelling's Trace & ,024 & 3,955 & ,008 & ,832 \\
\hline & Roy's Largest Root & ,024 & 3,955 & ,008 & ,832 \\
\hline \multirow[t]{4}{*}{ LIQUIDEZ } & Pillai's Trace & ,048 & 8,369 & ,000 & ,993 \\
\hline & Wilks' Lambda & ,952 & 8,369 & ,000 & ,993 \\
\hline & Hotelling's Trace & ,050 & 8,369 & ,000 & ,993 \\
\hline & Roy's Largest Root &, 050 & 8,369 &, 000 & ,993 \\
\hline
\end{tabular}


Tabela 3: Significância das Categorias (categoria x categoria)

\begin{tabular}{|c|c|c|c|c|c|}
\hline Categoria & Teste & Value & $\mathbf{F}$ & Sig. & Obs. Power \\
\hline \multirow[t]{4}{*}{ TIPO * REGULA } & Pillai's Trace & ,006 & 1,068 & ,362 & 290 \\
\hline & Wilks' Lambda & ,994 & 1,068 & ,362 & 290 \\
\hline & Hotelling's Trace & ,006 & 1,068 & ,362 & ,290 \\
\hline & Roy's Largest Root & ,006 & 1,068 & ,362 & 290 \\
\hline \multirow[t]{4}{*}{ TIPO $*$ CONTROLE } & Pillai's Trace &, 005 & ,911 &, 435 & 251 \\
\hline & Wilks' Lambda & ,995 & ,911 &, 435 & 251 \\
\hline & Hotelling's Trace &, 005 & ,911 & ,435 & 251 \\
\hline & Roy's Largest Root & ,005 & ,911 &, 435 & 251 \\
\hline \multirow{4}{*}{$\begin{array}{l}\text { REGULA * } \\
\text { CONTROLE }\end{array}$} & Pillai's Trace &, 024 & 4,137 &, 007 &, 850 \\
\hline & Wilks' Lambda & ,976 & 4,137 &, 007 &, 850 \\
\hline & Hotelling's Trace &, 025 & 4,137 &, 007 &, 850 \\
\hline & Roy's Largest Root &, 025 & 4,137 &, 007 & 850 \\
\hline \multirow[t]{4}{*}{ TIPO * LIQUIDEZ } & Pillai's Trace & ,009 & 1,589 & ,191 & 419 \\
\hline & Wilks' Lambda & ,991 & 1,589 & ,191 & ,419 \\
\hline & Hotelling's Trace & ,010 & 1,589 & ,191 & ,419 \\
\hline & Roy's Largest Root &, 010 & 1,589 & ,191 & ,419 \\
\hline \multirow{4}{*}{$\begin{array}{l}\text { REGULA * } \\
\text { LIQUIDEZ }\end{array}$} & Pillai's Trace & ,003 & ,431 & ,731 & , 136 \\
\hline & Wilks' Lambda & ,997 & ,431 &, 731 & , 136 \\
\hline & Hotelling's Trace & ,003 &, 431 & ,731 & , 136 \\
\hline & Roy's Largest Root &, 003 & 431 & ,731 & 136 \\
\hline \multirow{4}{*}{$\begin{array}{l}\text { CONTROLE * } \\
\text { LIQUIDEZ }\end{array}$} & Pillai's Trace &, 014 & 2,294 &, 077 & ,578 \\
\hline & Wilks' Lambda & ,986 & 2,294 & ,077 & ,578 \\
\hline & Hotelling's Trace &, 014 & 2,294 & ,077 & ,578 \\
\hline & Roy's Largest Root & ,014 & 2,294 & ,077 & ,578 \\
\hline
\end{tabular}

Tabela 4: Significância das Categorias (categoria x categoria x categoria)

\begin{tabular}{|c|c|c|c|c|c|}
\hline Categoria & Teste & Value & $\mathbf{F}$ & Sig. & Obs. Power \\
\hline \multirow{4}{*}{$\begin{array}{l}\text { TIPO * REGULA } \\
* \text { LIQUIDEZ }\end{array}$} & Pillai's Trace & ,014 & 2,411 & ,066 & ,602 \\
\hline & Wilks' Lambda & ,986 & 2,411 & ,066 &, 602 \\
\hline & Hotelling's Trace & ,014 & 2,411 & ,066 & ,602 \\
\hline & Roy's Largest Root &, 014 & 2,411 & ,066 &, 602 \\
\hline \multirow{4}{*}{$\begin{array}{l}\text { TIPO * REGULA } \\
* \text { CONTROLE }\end{array}$} & Pillai's Trace & ,013 & 2,244 & ,082 & ,567 \\
\hline & Wilks' Lambda & ,987 & 2,244 & ,082 & ,567 \\
\hline & Hotelling's Trace &, 013 & 2,244 & ,082 & ,567 \\
\hline & Roy's Largest Root & ,013 & 2,244 & ,082 & ,567 \\
\hline \multirow{4}{*}{$\begin{array}{l}\text { TIPO * } \\
\text { CONTROLE * } \\
\text { LIQUIDEZ }\end{array}$} & Pillai's Trace & ,007 & 1,203 & ,308 & ,323 \\
\hline & Wilks' Lambda & ,993 & 1,203 & ,308 & ,323 \\
\hline & Hotelling's Trace & ,007 & 1,203 & ,308 & ,323 \\
\hline & Roy's Largest Root & ,007 & 1,203 & ,308 & ,323 \\
\hline \multirow{4}{*}{$\begin{array}{l}\text { REGULA * } \\
\text { CONTROLE * } \\
\text { LIQUIDEZ }\end{array}$} & Pillai's Trace & ,001 & ,222 & ,881 & ,092 \\
\hline & Wilks' Lambda & ,999 & ,222 & ,881 &, 092 \\
\hline & Hotelling's Trace & ,001 & ,222 & ,881 &, 092 \\
\hline & Roy's Largest Root & ,001 & 222 & ,881 & ,092 \\
\hline
\end{tabular}


De acordo com os valores apresentados na tabela 2, observa-se que entre o conjunto de variáveis analisadas, somente as variáveis "controle acionário" e "liquidez" apresentaram significância estatística. Neste caso, os testes indicam que as médias dos retornos anormais acumulados variam nas datas do evento quando recebem o tratamento das variáveis "controle acionário" e "liquidez".

\subsection{INFLUÊNCIA DAS CARACTERÍSTICAS SOBRE AS MÉDIAS:}

Num segundo momento, as análises das contribuições de cada categoria sobre as médias dos retornos anormais estão apresentados nas tabelas 5, 6 e 7 . Nesta verificação procura-se rejeitar a hipótese nula, as variáveis independentes não contribuem para a diferenciação das médias dos retornos anormais acumulados em cada data de observação da janela do evento.

Tabela 5: Significância de cada variável sobre cada data de observação

\begin{tabular}{|l|c|c|c|c}
\hline \multicolumn{1}{c}{ Categoria } & $\begin{array}{c}\text { Dependent } \\
\text { Variable }\end{array}$ & $\mathbf{F}$ & Sig. & Obs Power \\
\hline TIPO & LNMENOS3 &, 003 &, 956 &, 050 \\
\cline { 2 - 5 } & LNZERO &, 097 &, 756 &, 061 \\
\cline { 2 - 5 } & LNMAIS3 &, 172 &, 679 &, 070 \\
\hline REGULA & LNMENOS3 &, 070 &, 791 &, 058 \\
\cline { 2 - 5 } & LNZERO &, 424 &, 515 &, 100 \\
\cline { 2 - 5 } & LNMAIS3 &, 082 &, 775 &, 059 \\
\cline { 2 - 5 } & LNMENOS3 & 7,851 &, 005 &, 799 \\
\cline { 2 - 5 } & LNZERO & 8,590 &, 004 &, 833 \\
\cline { 2 - 5 } & LNMAIS3 & 9,351 &, 002 &, 863 \\
\cline { 2 - 5 } & LNMENOSO & 18,240 &, 000 &, 989 \\
\cline { 2 - 5 } & LNZERO & 12,406 &, 000 &, 940 \\
\cline { 2 - 5 } & LNMAIS3 & 19,197 &, 000 &, 992 \\
\hline
\end{tabular}


Tabela 6: Significância de 2 variáveis sobre cada data de observação

\begin{tabular}{l|c|c|c|c}
\hline \multicolumn{1}{c|}{ Categoria } & $\begin{array}{c}\text { Dependent } \\
\text { Variable }\end{array}$ & $\mathbf{F}$ & Sig. & Obs Power \\
\hline \multirow{4}{*}{ TIPO * REGULA } & LNMENOS3 & 1,831 &, 177 &, 272 \\
\cline { 2 - 5 } & LNZERO &, 001 &, 971 &, 050 \\
\cline { 2 - 5 } & LNMAIS3 &, 016 &, 898 &, 052 \\
\hline TIPO * CONTROLE & LNMENOS3 & 1,243 &, 265 &, 199 \\
\cline { 2 - 5 } & LNZERO & 1,513 &, 219 &, 233 \\
\cline { 2 - 5 } & LNMAIS3 &, 006 &, 941 &, 051 \\
\hline REGULA * & LNMENOS3 & 9,135 &, 003 &, 855 \\
\cline { 2 - 5 } CONTROLE & LNZERO & 8,879 &, 003 &, 845 \\
\cline { 2 - 5 } & LNMAIS3 & 9,000 &, 003 &, 849 \\
\hline TIPO * LIQUIDEZ & LNMENOS3 & 1,134 &, 287 &, 186 \\
\cline { 2 - 5 } & LNZERO & 3,734 &, 054 &, 488 \\
\cline { 2 - 5 } & LNMAIS3 &, 238 &, 626 &, 078 \\
\hline REGULA * & LNMENOS3 &, 436 &, 509 &, 101 \\
\cline { 2 - 5 } & LNZERO &, 001 &, 970 &, 050 \\
\cline { 2 - 5 } & LNMAIS3 &, 221 &, 639 &, 076 \\
\hline CONTROLE * & LNMENOS3 & 2,308 &, 129 &, 329 \\
\cline { 2 - 5 } & LNZERO & 6,241 &, 013 &, 703 \\
\cline { 2 - 5 } & LNMAIS3 & 1,024 &, 312 &, 173
\end{tabular}

Tabela 7: Significância de 3 variáveis sobre cada data de observação

\begin{tabular}{l|c|c|c|c|}
\hline \multicolumn{1}{c|}{ Categoria } & $\begin{array}{c}\text { Dependent } \\
\text { Variable }\end{array}$ & F & Sig. & Obs Power \\
\hline TIPO * REGULA * & LNMENOS3 & 6,465 &, 011 &, 718 \\
\cline { 2 - 5 } CONTROLE & LNZERO & 4,071 &, 044 &, 521 \\
\cline { 2 - 5 } & LNMAIS3 & 1,769 &, 184 &, 264 \\
\hline \multirow{2}{*}{$\begin{array}{l}\text { LIPO * REGULA * } \\
\text { LIQUEZ }\end{array}$} & LNMENOS3 & 6,557 &, 011 &, 724 \\
\cline { 2 - 5 } & LNZERO & 5,111 &, 024 &, 617 \\
\cline { 2 - 5 } & LNMAIS3 & 2,078 &, 150 &, 301 \\
\hline TIPO * CONTROLE & LNMENOS3 & 2,331 &, 127 &, 332 \\
\cline { 2 - 5 } & LNZERO &, 614 &, 434 &, 122 \\
\cline { 2 - 5 } & LNMAIS3 & 2,171 &, 141 &, 313 \\
\hline REGULA * & LNMENOS3 &, 325 &, 569 &, 088 \\
\cline { 2 - 5 } $\begin{array}{l}\text { CONTROLE * } \\
\text { LIQUIDEZ }\end{array}$ & LNZERO &, 593 &, 442 &, 120 \\
\cline { 2 - 5 } & LNMAIS3 &, 101 &, 751 &, 062
\end{tabular}

Pelos dados apresentados nas tabelas 5, 6, e 7 observa-se que as variáveis "controle acionário" e "liquidez" rejeitam a hipótese nula ao nível de $5 \%$, contribuindo de forma significativa para explicar as diferenças entre as médias dos retornos anormais acumulados em todas as datas de observação que compõem a janela de evento estudada.

Os resultados obtidos também comprovam que as variáveis "tipo de ações" e "regulamentação" contribuem para explicar a diferença entre as médias dos retornos anormais acumulados, mas somente quando associadas as variáveis controle acionário e liquidez. 


\section{COMENTÁRIOS FINAIS}

Os resultados encontrados pelo teste empírico utilizado neste estudo possibilitaram a geração de algumas evidências em relação à hipótese de trabalho estruturada:

- As evidências encontradas indicam que algumas variáveis possuem a capacidade de contribuir para explicar as diferenças entre os retornos anormais em períodos em torno da data de divulgação das informações contábeis.

* Entre as variáveis estudadas, foram encontradas evidências de que as variáveis "controle acionário" e "liquidez" influenciam de forma significativa sobre a diferença entre as médias dos retornos anormais.

- As variáveis "tipo de ação" e "regulamentação" apresentaram capacidade de explicação, mas somente quando exercem a sua influência em conjunto com as outras variáveis "controle acionário" e "liquidez".

As evidências geradas por este estudo sustentam as questões que relacionam as influências de características dos ativos sobre a relevância das informações contábeis para o mercado de capitais. Porém, dadas as evidências encontradas nesta pesquisa, e o conjunto de conhecimento existe sobre a eficiência do mercado brasileiro, alguns pontos devem ser considerados:

- As evidências encontradas no trabalho convergem e divergem do conjunto de evidências encontradas pela literatura dedicada a estudar a relevância da contabilidade no mercado brasileiro.

- As evidências encontradas neste estudo corroboram os resultados de vários trabalhos no mercado brasileiro, indicando que algumas características interferem na reação das ações à divulgação das informações contábeis.

- Existem, porém, divergências quanto às variáveis que influenciam na reação. Os resultados deste estudo indicaram que o Tipo de Controle não tem a capacidade de influenciar a reação. Esta evidência diverge das encontradas por alguns trabalhos, como Lopes (2001) e Sarlo Neto (2005), que encontraram diferenças nas reações entre as ações preferenciais e ordinárias.

A partir dos resultados encontrados, podem-se sugerir algumas propostas para novas pesquisas, na tentativa de buscar novas evidências sobre a influência das características dos mercados sobre a reação dos preços à divulgação da informação contábil. Entre as sugestões, encontram-se: a ampliação da janela do evento para analisar a influência de características sobre períodos superiores a três meses; a verificação de outras características, além das estudadas nesta pesquisa (exemplo: valor de mercado, risco, endividamento etc...); e a aplicação de outras técnicas estatísticas, tais como análise de cluster ou regressão logística.

\section{REFERÊNCIAS}

BALL, R. J., BROWN, W. An empirical evaluation of accounting income numbers. Journal of Accounting Research, v. 6, p.159-178, Autumn, 1968.

BALL, R. K., WATTS, R. S. P. Economic Determinants of the relation between earnings changes and stock returns. The Accounting Review, v. 68, n. 3, July, p. 622-638, 1993. 
BEAVER, W. H., CLARKE, R., WRIGHT, W. The Association Between Unsystematic Security Returns and the Magnitude of Earnings. Forecast Errors. Journal of Accounting Research, 17, Autumn, p. 316-340, 1979.

BEAVER, W. H. Financial reporting: an accounting revolution. $2^{\text {nd }}$ ed. Englewood Cliffs.: Prentice hall, 1998.

BERNARDO, H. P. Avaliação empírica do efeito dos anúncios trimestrais do resultado sobre o valor das ações no mercado brasileiro de capitais - um estudo de evento. 2001. 157f. Dissertação (Mestrado em Ciências Contábeis) - Departamento de Contabilidade e Atuária - Faculdade de Economia, Administração e Contabilidade - Universidade de São Paulo, São Paulo. 2001.

BROWN, P. The Impact of the Annual Net Profit Report on the Stock Market. The Australian Accountant, July, p. 277-283, 1970.

CORRAR, L. J. THEOPHILO, C. R. (Coords.). Pesquisa Operacional para decisão em contabilidade e administração: contabilometria. São Paulo: Atlas, 2004.DAVIS-FRIDAY, P., RIVERA, J. Inflation Accounting and 20-F Disclosures: Evidence from Mexico. Accounting Horizons, v. 14, n. 2, 2000.

GRANT, E. B. Market Implications of Differential Amounts of Interim Information. Journal of Accounting Research, 18, Spring, p. 255-268, 1980.

GUIMARÃES, S. F., NESS, W. L. Desempenho Relativo de Ações Ordinárias e Preferenciais. In: Encontro Brasileiro de Finanças, 1. Anais do $1^{\circ}$ Encontro Brasileiro de Finanças, São Paulo, 2001.

FAMA E. F. Efficient Capital Markets: II. The Journal of Finance, v. 46, 5, p. 1875-1617, December, 1991.

HAIR, J. F., ANDERSON, R. E., TATHAM, R. L., BLACK, W. C. Análise Multivariada de Dados. 5.ed. Porto Alegre: Bookman, 2005. HENDRIKSEN, E. S., VAN BREDA, M. F. Teoria da Contabilidade; Tradução de Antonio Zoratto Sanvincente. São Paulo: Atlas, 1999.

IUDÍCIBUS, S. de. Teoria da Contabilidade. 6. ed. São Paulo: Atlas, 2000.

LOPES, A. B. A Informação Contábil e o Mercado de Capitais. São Paulo: Pioneira Thomson Learning, 2002.

LOPES, A. B. A relevância da informação contábil para o mercado de capitais: o Modelo de Ohlson aplicado à Bovespa. 2001. 308f. Tese (Doutorado em Controladoria e Contabilidade) - Departamento de Contabilidade e Atuária, Faculdade de Economia, Administração e Contabilidade, Universidade de São Paulo. São Paulo, 2001.

MACKINLEY, A. C. Event Studies in Economics and Finance. Journal of Economics Literature, v. 35, 1, p. 13-39, March, 1997.

MAROCO, João. Análise Estatística. 2. ed. Lisboa: Sílabo, 2003.PARK, C. W. PINCUS, M. Internal versus external equity funding sources and earnings response coeficients. Working Paper. Department of Accounting, The University of lowa. June, 1997.

PELLICER, M. J. S. REES, W. P. Regularities in the equity price response to earnings announcements in Spain. Working Paper Series, Department of Accounting \& Finance. University of Glasgow. September, 1999.

PEROBELLI, F. F. C., NESS, W. L. Reações do Mercado Acionário a Variações Inesperadas nos Lucros das Empresas: um Estudo sobre a Eficiência Informacional no Mercado Brasileiro. In: ENANPAD 2000, 24. Anais do XXIV Encontro da Anpad, Florianópolis, 2000. 
PROCIANOY, J. L.; CASELINI, C. N. A emissão de ações como fonte de crescimento ou como fator de redução do risco financeiro: resultados empíricos. Revista de Administração. 1997.

, ANTUNES, M. A. Os Efeitos das Decisões de Invetimento das Empresas sobre o preço de suas ações no mercado de capitais. In: ENANPAD 2001, 25. Anais do XXV Encontro da Anpad, Campinas, 2001.

SARLO NETO,A. LOPES, A.B. O impacto da regulamentação sobre a relação entre lucro e retorno das ações das empresas dos setores elétrico e financeiro no Brasil. In: ENANPAD 2002, 26. Anais do XXVI Encontro da Anpad, Salvador, 2002.

SARLO NETO, A. A Reação dos Preços à Divulgação dos Resultados Contábeis: Evidências Empíricas sobre a Capacidade Informacional da Contabilidade no Mercado Acionário Brasileiro. 2004, 243f. Dissertação (Mestrado em Ciências Contábeis) - FUCAPE - Fundação Capixaba de Pesquisa em Economia, Contabilidade e Finanças. Vitória, 2004.

., LOSS, L., NOSSA, V. e TEIXEIRA, A. J. C. Uma Investigação sobre a Capacidade Informacional dos Lucros Contábeis no Mercado Acionário Brasileiro. In: Congresso USP de Controladoria e Contabilidade, 3, 2003, São Paulo (SP). Anais do $3^{\circ}$ Congresso USP de Controladoria e Contabilidade em CD-ROM, 2003.

TERRA, P. R. O Valor de Mercado do Controle de Companhias Abertas: Evidências Empíricas dos Custos e Benefícios dos Direitos de Voto das Ações no Brasil. 1993. Dissertação (Mestrado em Administração). Faculdade de Ciências Econômicas. Porto Alegre: Universidade Federal do Rio Grande do Sul, 1993.

WATTS, R. L., ZIMMERMAN, J. L. Positive Accounting Theory. New Jersey: Princete-Hall, 1986.

WASSERMAN, Cláudio. MANOVA. Seminário e notas de aula, Contabilometria. Outubro, 2005.

\section{ENDEREÇO DOS AUTORES}

Centro de Ciências Jurídicas e Econômicas

Universidade Federal do Espírito Santo

Campus Universitário Alaor Queiroz de Araújo

Av. Fernando Ferrari, s/ número - Goiabeiras

Vitoria, ES - Brasil

29060-900

Fundação Instituto Capixaba de Pesquisa em Contabilidade, Economia e Finanças Av. Fernando Ferrari, 1358 - Goiabeiras

Vitoria, ES - Brasil

29075-010

\footnotetext{
i Publicados nos principais congressos e revistas
} 
i A data de divulgação não compreende a data de publicação, na qual as demonstrações são efetivamente publicadas em algum meio impresso de comunicação conforme a legislação. A data de divulgação considerada na pesquisa é aquela em que as demonstrações contábeis são disponibilizadas ao mercado.

iii Cálculo do Retorno Anormal Mensal:

Para esta pesquisa foi selecionado o modelo estatístico conhecido como Modelo de Mercado. Esse modelo estatístico foi o mesmo utilizado por Ball e Brown (1968), e também amplamente utilizado em estudos de eventos. Sendo o retorno iil da ação calculado da seguinte forma:

$$
r_{i, t}=\frac{P_{t}-P_{t-1}}{P_{t-1}}
$$

Em que: ri,t =taxa de retorno da ação i no período [t,t-1], Pi,t = preço da ação i no mês t e Pi,t1 = preço da ação i no mês $\mathrm{t}-1$.

Para o cálculo do retorno anormal será utilizado o Modelo de Mercado, que consiste em um modelo estatístico representado por uma regressão linear entre os retornos $r_{\mathrm{i}}$ de uma determinada ação e os retornos da carteira do mercado $r_{\mathrm{m}}$.

$$
r_{i, t}=\alpha_{i}+\beta_{i} r_{m, t}+e_{i, t}
$$

Em que: $r_{i, t}=$ Retorno da empresa $i$ no período $t, \alpha_{i}=$ Interceptor da empresa $i, \beta_{i}=$ Coeficiente de variação da empresa $i$ no período $t, \mathrm{r}_{\mathrm{m}, \mathrm{t}}=$ Retorno do mercado no período $t$, e $\mathrm{e}_{i, t}=$ Erro da empresa $i$ no período $t$.

Por meio dos estimadoresiii calculados pelo modelo de mercado, podem-se calcular os retornos esperados para uma ação em um determinado período de acordo com a seguinte equação:

$$
E\left(r_{i, t}\right)=\alpha_{i}+\beta_{i} r_{m, t}
$$

Em que:E $\left(r_{i, t}\right)=$ Retorno esperado da empresa i no período $t, \alpha_{i}=$ Interceptor da empresa $i, \beta_{i}$ $=$ Coeficiente de variação da empresa $i$ no período $t, \mathrm{e} r_{\mathrm{m}, \mathrm{t}}=$ Retorno do mercado no período $t$.

A diferença entre o retorno esperado $E\left(r_{i, t}\right)$ pelo Modelo de Mercado e o retorno da ação $r_{i t}$ em um determinado período é denominada de retorno anormal. Depois de calcular o retorno esperado pelo Modelo de Mercado, encontra-se o retorno anormal da seguinte forma:

$$
R A_{i t}=r_{i t}-E\left(r_{i, t}\right)
$$

Em que: $R A=$ Retorno Anormal para a empresa $i$ no período $t, r_{i t}=$ Retorno da empresa $i$ no período $t$, e $E\left(r_{i, t}\right)=$ Retorno esperado da empresa i no período t.

" MANOVA é uma técnica de dependência que compara as diferenças de médias para duas ou mais variáveis dependentes métricas, com base em um conjunto de variáveis independentes categóricas (não-métricas)

${ }^{v} \mathrm{O}$ Lucro por ação: $\mathrm{LPA} i, t=\mathrm{LL} i, t / \mathrm{ni}, t$ onde: $\mathrm{LL}_{i, t}=$ Lucro Líquido da empresa $i$ no período $t, \mathrm{n}_{i . t}=\mathrm{N}$ o ações em circulação da empresa $i$ em $t$.

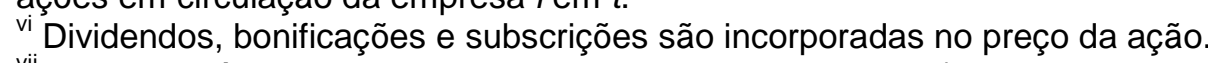

vii Algumas séries de preços apresentaram-se incompletas (algumas ações não registraram cotações em todos os dias de todos os meses do período analisado). Nesses casos, as séries foram completadas por intermédio da ineterpolação Linear.

viii Para avaliar a autocorrelação dos resíduos foi utilizada a estatística de Durbin-Watson

${ }^{\text {ix }}$ Desconsiderou-se a presença da Heterocedasticidade em alguns eventos. 